\title{
Contents, Vol. 68, 1929
}

\section{Inhalts-Verzeichnis.}

E $1 / 8$ enarbeiten.

Seite

Barczinski, S., Ein Fall von Ophthalmomyiasis interna anterior (Behr) 353

Birch-Hirschieíd, A., Hochgradige Kurzsichtigkeit und

künstliche Unterbrechung der Schwangerschaft . . 127 Fazakas, A., Über eine neue Methode der Denigschen

Transplantation zur Pannus-Therapie 356

Fuchs, A., Über mehrkernige Plasmazellen und das besondere Vorkommen von Russelschen Körperchen . 134 Golowin, S.S., Über den Fortschritt in der Ophthalmologie und ihre künftigen Errungenschaften 33

Gutzeit, R., Über familiäre knötchenförmige Hornhauttrübung $\quad 349$

Hamann, J., Beitrag zur Diagnose und Therapie von Hypophysentumoren 317

Hollos, L. und Linksz, A. Über die Behandlung von Horn-

hauterkrankungen mit ultraviolettem Licht .... 152 Junius, P., Bemerkungen zum Krankheitsbild der , Reti-

nitis” (Coats), der „Netzhautdegeneration mit multiplen

Aneurysmen" (Th. Leber) und der sog. ,,Angiomatosis

retinae" (Czermak-von Hippel) 207

Krasso, J., Über die Möglichkeit der Beeinflussung des

Glaukoms durch die Röntgenbestrahlung der Schild-

drüse 163

Krause, K., Naevus flammeus und Glaukom 244

Linksz, A., siehe Hollos, L.

Mandicevski, V., Über eine besondere Art der Kalkablage-

rung im Auge 221

Meves, H., In vivo nachgewiesene verkalkte Linse . . 30 Mussa-Beili, Uminissa-Chanum, Kasuistik der Korektopie 20 -, - Ein Fall von Lähmung des Nervus abducens nach

Grippe . $\quad 361$

Preßburger, E., Über die zystoide Entartung der Netz-

hautperipherie 331

Reimers, 0., Zur Verwendung yon Fascia lata am Auge 27 Rieger, H., und Trauner, R., Über einen Fall von Biedl-

Bardetschem Syndrom und die Erblichkeitsverhält

nisse dieses Zustandes235 
Rönne, H., Die Diagnostik des inflammatorischen Exoph-

thalmus bei akuten Infektionskrankheiten .... 1 Saltier, C. H., Gerät zür objektiven und subjektiven

Messung des Schielwinkels, zur graphischen Dar-

stellung des Blickfeldes und zur Gesichtsfeldunter-

suchung nach Bjerrum 345

Schindler, E., Über das Auftreten persistierender Desce-

metozelen (hierzu Tafel I) 22

Sommer. J., Über einen histologischen Befund in einem

Falle von tiefer Keratitis

.... 260

Trauner, R., siehe Rieger, H.

IV -

Aus der Praxis für die Praxis.

Seite

Awguschewüsch, P., Echinokokkus der Augenhöhle . . 364 Luftmann, A., Eine Modifikation des

Axenfeldschen Ver-

fahrens bei Ausschneidung der unteren Tränendrüse 39

Berichte über die deutsehe ophthalmologische Literatur.

Untersuchungsmethoden. I. und II. Semester 1928. Von

Doz. Dr. R. Kramer in Wien 41

Spezielle Pathologie und Therapie im Jahre 1926. Von

Dr. E. Kunz in Königsberg 268

Berichte über die ausländische ophthalmologische Literatur.

Bericht über die italienische ophthalmologische Literatur1926. Von Dr. C. Mariottí in Bologna und Dr. E. Guz-mann in Wien $\quad 53,179$

Gesellschaftsberichte.

Ophthalmologische Gesellschaft in Wien. Sitzung vom 18. März 192984

Sitzung vom 15. April 1929190

Sitzung vom 27. Mai $1929 \quad 304$

Sitzung vom 17. Juni 1929366

Berliner augenärztliche Gesellschaft. Sitzung vom 28. Februar $1929 \quad 94$

Sitzung vom 25. April 1929. 194

Sitzungen vom 30. Mai und 27. Juni 1929 370Bericht über die 16. Sitzung der

Ophthalmologischen Abteilungder Gesellschaft für Wissenschaft und Leben im rheinisch-

westfälischen Industriebezirk am 24. November 1928 ... 9813. Tagung der Deutschen

Ophthalmologischen Gesellschaft in derTschechoslowakischen Republik, gemeinsam mit der

Ver-einigung südostdeutscher Augenärzte, in Prag am 23. und

24. Februar 1929

104

Verein der Augenärzte von Ost- und Westpreußen. Sitzung vom

24. November 1928

195

54. Versammlung Rhein.-westfälischer Augenärzte. Sitzung vom

10. März 1929 298

Augenärztliche Gesellschaft in Hamburg. W.-S. 1928/29 ....306Vereinigte Medizinische

Gesellschaft in Kiew, Sektion für Augen-

heilkunde. Sitzung vom 18. Oktober 1928

Sitzung vom 8. Dezember 1928

118 
Sitzung vom 23. Dezember 1928

Sitzungen vom 6. Febr., 20. Febr. u. 6. März 1929 310Jubiläumskongreß der Ungarischen ophthalmologischen Gesellschaft am 25. und 26. Mai 1929 . .

373

Diagnose und Therapie $119,201,313,377$

Unfall- und Versicherungskunde 204, 377

Blindenwesen

122

Buchbesprechungen 205, 379

Personalien

316

S. Sachregister

382

Namenregister

390 